\title{
How dust fragmentation may be beneficial to planetary growth by pebble accretion
}

\author{
J. Drążkowska ${ }^{1}$, S. M. Stammler ${ }^{1}$, and T. Birnstiel ${ }^{1,2}$ \\ ${ }^{1}$ University Observatory, Faculty of Physics, Ludwig-Maximilians-Universität München, Scheinerstr. 1, 81679 Munich, Germany \\ e-mail: joanna.drazkowska@lmu.de \\ 2 Exzellenzcluster ORIGINS, Boltzmannstr. 2, 85748 Garching, Germany
}

Received 17 November 2020 / Accepted 21 December 2020

\begin{abstract}
Context. Pebble accretion is an emerging paradigm for the fast growth of planetary cores. Pebble flux and pebble sizes are the key parameters used in the pebble accretion models.

Aims. We aim to derive the pebble sizes and fluxes from state-of-the-art dust coagulation models and to understand their dependence on disk parameters and the fragmentation threshold velocity, and the impact of those on planetary growth by pebble accretion.

Methods. We used a 1D dust evolution model including dust growth and fragmentation to calculate realistic pebble sizes and mass flux. We used this information to integrate the growth of planetary embryos placed at various locations in the protoplanetary disk. Results. Pebble flux strongly depends on disk properties including size and turbulence level, as well as the dust aggregates' fragmentation threshold. We find that dust fragmentation may be beneficial to planetary growth in multiple ways. First of all, it prevents the solids from growing to very large sizes, at which point the efficiency of pebble accretion drops. What is more, small pebbles are depleted at a lower rate, providing a long-lasting pebble flux. As the full coagulation models are computationally expensive, we provide a simple method of estimating pebble sizes and flux in any protoplanetary disk model without substructure and with any fragmentation threshold velocity.
\end{abstract}

Key words. accretion, accretion disks - planets and satellites: formation - protoplanetary disks - methods: numerical

\section{Introduction}

In the classical paradigm of planet formation, a significant fraction of solids is very quickly converted into kilometer-sized planetesimals. Some of those planetesimals continue to grow rapidly via runaway growth (e.g., Wetherill \& Stewart 1989; Ida \& Makino 1993; Kokubo \& Ida 1996). However, this fast stage of growth is soon over as the embryo starts to stir its feeding zone and the accretion transitions to an oligarchic growth stage, slowing down with embryo mass (e.g., Kokubo \& Ida 1998; Ormel et al. 2010). By the end of the oligarchic growth, the embryo absorbs all of the material in its immediate vicinity and reaches the isolation mass, which is as low as Mars mass in the terrestrial planet region, but it increases with distance from the star (Kokubo \& Ida 2002). The isolation mass may be high enough to reproduce the cores of giant planets, but the core growth timescale in the planetesimal-driven scenario becomes prohibitively long to allow for the accretion of a gaseous atmosphere outside of the Jupiter location (Thommes et al. 2002, 2003; Levison et al. 2010; Johansen \& Bitsch 2019).

These drawbacks of the classical planetesimal-driven model motivated the development of the alternative "pebble accretion" scenario. In this paradigm, the embryo accretes centimeter-sized pebbles rather than planetesimals. Gravity and gas drag act together to enhance the cross-section of a planetary embryo for its encounters with pebbles, and thus speeds up the growth (Ormel \& Klahr 2010; Lambrechts \& Johansen 2012). Because pebbles are rapidly drifting through the protoplanetary disk, the size of the feeding zone increases, allowing the embryos to grow to larger sizes before the pebble flux is halted by planet-disk interactions (Lambrechts et al. 2014).
In the pebble accretion scenario, the outcome of planet formation is determined by the sizes and flux of the pebbles drifting through the protoplanetary disk. Kretke \& Levison (2014) used large pebbles with a size distribution between 0.5 and $5 \mathrm{~m}$ (corresponding to the Stokes number of 0.25 and 25) and a constant pebble flux of $0.13 M_{\oplus} \mathrm{yr}^{-1}$. They found that reproducing the giant planets in the Solar System is challenging, as pebble accretion tends to convert too many planetesimals into large embryos. Levison et al. (2015a) showed that this difficulty may be mitigated if pebbles form gradually so that the growing planetesimals have time to interact and stir each other. Recently, Lambrechts et al. (2019) showed that a high pebble flux leads to the formation of giant planets, while a lower flux leads to the formation of super-Earths. However, for simplicity many authors studying the pebble-driven planetary growth define a fixed size or Stokes number of pebbles and the value of the pebble flux (or the pebble-to-gas flux ratio) as arbitrary parameters (typically fixed in orbital distance and/or time), independently of the protoplanetary disk model (Johansen et al. 2015, 2019; Liu et al. 2019; Lambrechts et al. 2019; Ogihara \& Hori 2020; Brasser \& Mojzsis 2020; Wimarsson et al. 2020).

Lambrechts \& Johansen (2014) presented an algorithm for calculating the pebble flux and pebble Stokes number, which is often used by the community (Levison et al. 2015a,b; Ida et al. 2016; Bitsch \& Johansen 2016; Matsumura et al. 2017; Brügger et al. 2018; Bitsch 2019; Bitsch et al. 2019a; Izidoro et al. 2019). In this model, which is based on a simplified scenario of dust evolution, it is assumed that all dust grains grow until the growth timescale becomes longer than the drift timescale. Since the growth timescale strongly increases with distance, the "pebble formation front" moves outward, leading to subsequent portions 
of solids decoupling from the gas and maintaining inward pebble flux until the front reaches the outer edge of the disk. This picture is roughly consistent with the outcome of dust coagulation models, as long as dust growth can proceed without being hindered by fragmentation. However, the increase of collision speeds with aggregate sizes often prevents dust growth from reaching those sizes.

Laboratory experiments show that collisions of dust aggregates already become destructive at speeds much lower than predicted for the protoplanetary disk environment. The exact fragmentation threshold speed remains uncertain, and it was shown to strongly depend on the porosity and composition of the aggregates (Wada et al. 2011; Meru et al. 2013; Blum 2018). Until recently, it was believed that the icy aggregates outside of the water ice line were significantly more sticky than the dry aggregates inside of the ice line (Aumatell \& Wurm 2014; Gundlach \& Blum 2015). However, newer laboratory data do not support this view, demonstrating that the icy aggregates break easily (Gundlach et al. 2018; Steinpilz et al. 2019; Musiolik \& Wurm 2019).

Nevertheless, most of the works concerned with planetary growth via pebble accretion published to date neglect the effect that the fragmentation of pebbles has on their sizes and flux (the exceptions are Chambers 2016, 2018, Guilera et al. 2020, and Venturini et al. 2020a,b). In this paper, for the first time, we study the growth of a planetary embryo by pebble accretion in connection with a self-consistent dust evolution model, considering the full size distribution obtained in a detailed dust coagulation simulation.

This paper is organized as follows. We present the numerical model used in this work in Sect. 2. We present the resulting planetary growth tracks and their dependence on the parameters that are the most important to defining the dust evolution outcome in Sect. 3. We present a simple way of predicting a realistic pebble flux and sizes that is valid in both fragmentation-dominated and drift-dominated protoplanetary disks in Sect. 4. In Sect. 5, we discuss our work, and we summarize it in Sect. 6.

\section{Model}

\subsection{Protoplanetary disk model}

We modeled the protoplanetary disk surrounding a solar-mass star with an effective temperature of $T_{\text {eff }}=5772 \mathrm{~K}$. In the initial condition, we set the gas surface density as a function of the radial distance $r$ to the often used, self-similar solution to the viscous evolution equations (Lynden-Bell \& Pringle 1974):

$\Sigma_{\text {gas }, 0}=\frac{M_{\text {disk }}}{2 \pi r_{\mathrm{c}}^{2}}\left(\frac{r}{r_{\mathrm{c}}}\right)^{-1} \exp \left(-\frac{r}{r_{\mathrm{c}}}\right)$,

where $M_{\text {disk }}$ is the initial disk mass, and $r_{\mathrm{c}}$ is the characteristic radius and the dust surface density to

$\Sigma_{\text {dust }, 0}=Z \cdot \Sigma_{\text {gas }, 0}$,

where $Z$ is the global solid-to-gas ratio. In all the models presented in this paper, we considered disks with exactly the same initial mass budget. $M_{\text {disk }}$ is set to $0.2 M_{\odot}$, and $Z$ has the standard value of 0.01 , which corresponds to the initial mass in solids of about $650 M_{\oplus}$. We considered two values of the characteristic radius, $r_{\mathrm{c}}=30 \mathrm{au}$, in the compact disk, and $r_{\mathrm{c}}=300 \mathrm{au}$ in the large disk model. The initial gas surface density profiles are presented in the upper panel of Fig. 1. Despite their high mass, both
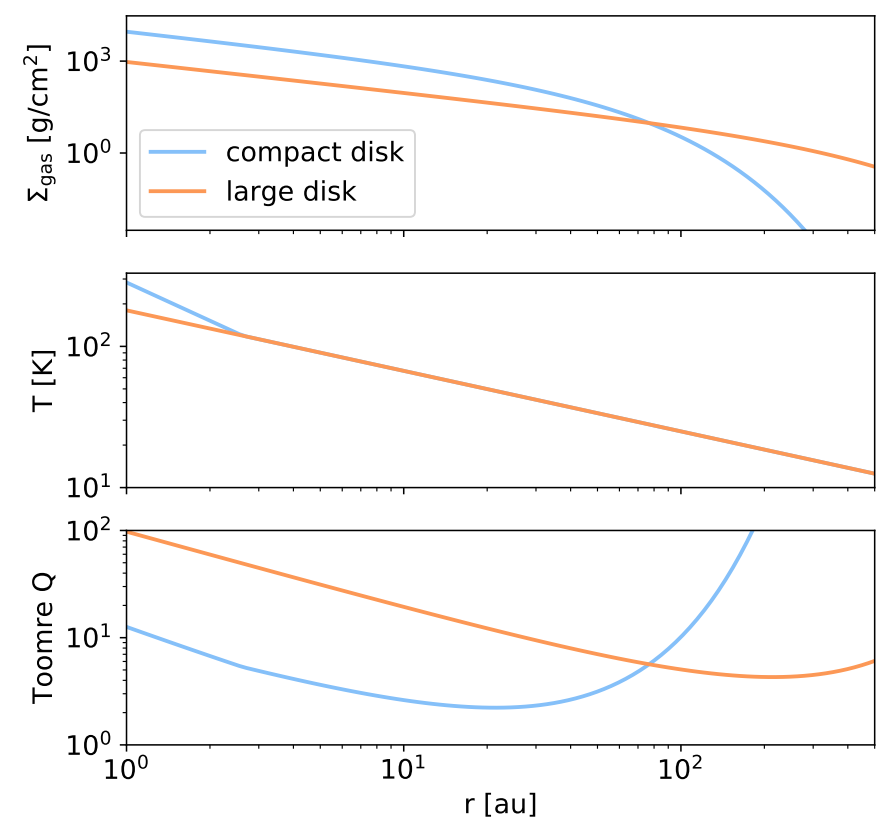

Fig. 1. Comparison of compact and large disk models with $\alpha=10^{-4}$. Top: gas surface density profiles. Middle: temperature in disk midplane. Bottom: Toomre $Q$ parameter.

of the disk models are gravitationally stable, with the Toomre $Q>2$, as shown in the bottom panel of Fig. 1 .

The gas disk evolves viscously, which means that the characteristic radius increases, while the surface density drops with time. The speed of viscous evolution is determined by the $\alpha$ parameter. We set the default value to $\alpha=10^{-4}$. In Sect. 3.3, we show the effects of varying the $\alpha$ parameter. In this work, we do not consider disk dispersal by photoevaporation.

The pebble accretion rate is sensitive to the vertical scale height of gas $H_{\text {gas, }}$ and thus to the disk's temperature structure. In this paper, we take into account both stellar irradiation and viscous heating using the simple prescription proposed by Ida et al. (2016, in their Eqs. (7) and (8)). Most of the disk is heated by stellar irradiation, leading to a shallow temperature profile of $T_{\text {irr }} \propto r^{-3 / 7}$. In the inner part of the disk, viscous heating may change the temperature profile to a steeper function of the radial distance, $T_{\text {vis }} \propto r^{-9 / 10}$. In the viscous heating regime, the temperature depends on the gas mass accretion rate, and thus this effect is particularly important in the compact disk, as is visible in the middle panel of Fig. 1, and in models with $\alpha=10^{-3}$, where the gas mass flux, defined as $\dot{M}_{\text {gas }}=3 \pi \alpha \Sigma_{\text {gas }} H_{\text {gas }}^{2} \Omega_{\mathrm{K}}$, with $\Omega_{\mathrm{K}}$ being the Keplerian frequency, is higher.

\subsection{Dust evolution}

We investigated the dust evolution on a logarithmic radial grid with 120 grid points spaced between 1 and 1000 au. We computed dust evolution in the azimuthally and vertically averaged framework using the DustPy code, a Python-based version dust coagulation model similar to the code presented by Birnstiel et al. (2010). At every radial distance to the star, a dust mass grid with nine mass bins per mass decade was built, and the Smoluchowski equation was used to solve for dust growth and fragmentation. We assume that the dust grains initially have radii of one micron and internal densities of $1.25 \mathrm{~g} \mathrm{~cm}^{-3}$. The collisions were driven by the Brownian motion and turbulence, which was prescribed with the $\alpha$ parameter, radial and azimuthal drift, 

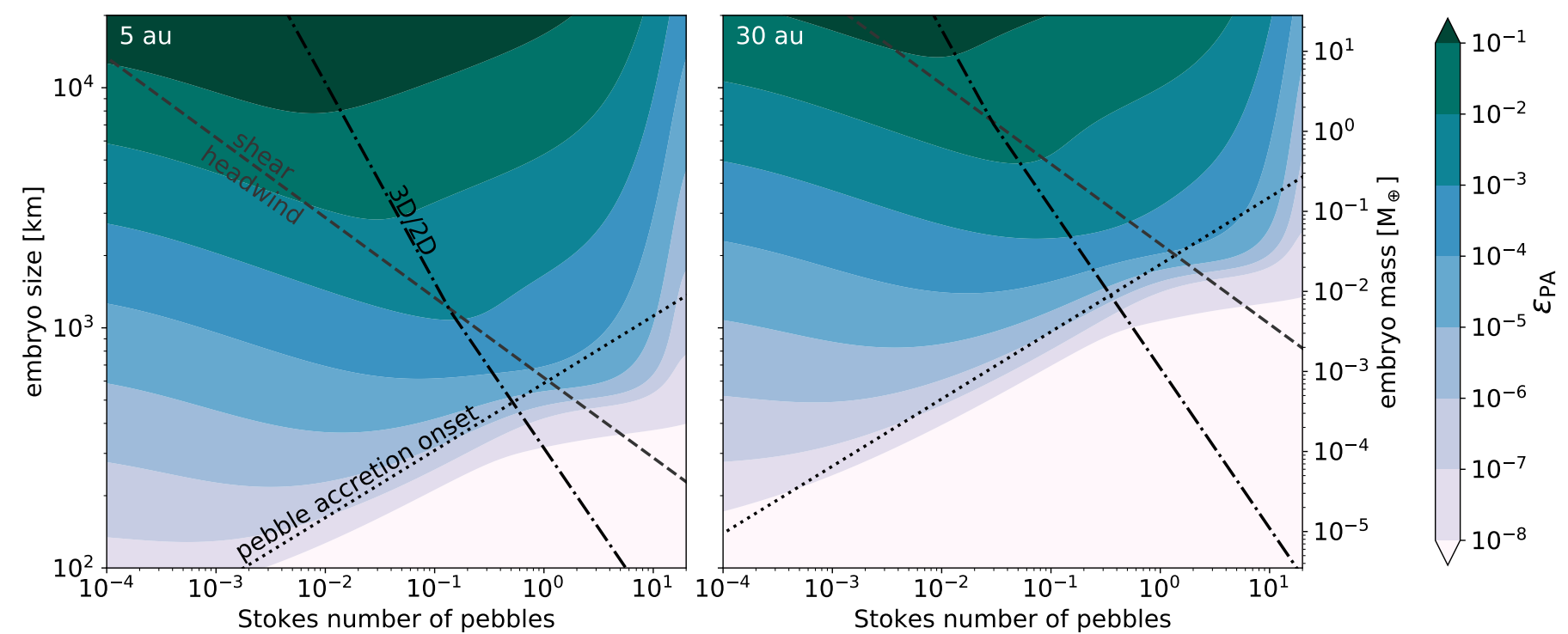

Fig. 2. Efficiency of pebble accretion parameter, $\varepsilon_{\mathrm{PA}}$, as a function of the planetary embryo's size and pebble's Stokes number calculated at 5 (left panel) and at 30 au (right panel) in the compact disk model with $\alpha=10^{-4}$. The dotted line corresponds to the pebble accretion onset (when the aerodynamic effects become important). The gray, dashed line shows the transition between the headwind and shear regimes of pebble accretion. The dashed-dotted line shows the transition between 3D and 2D pebble accretion.

and dust settling. We considered various values of the fragmentation threshold speed $v_{\mathrm{f}}$, between $1 \mathrm{~m} \mathrm{~s}^{-1}$ and $\infty$, corresponding to no fragmentation at all. In line with the newest laboratory results, we did not employ a transition at the water ice line, but we did use a single value of $v_{\mathrm{f}}$ independent on the radial distance.

The radial drift of particles in every mass bin, $i$, was computed based on their local Stokes number (Weidenschilling 1977) $\mathrm{St}_{i}$ :

$v_{\mathrm{r}, i}=\frac{2 v_{\eta} \mathrm{St}_{i}}{1+\mathrm{St}_{i}^{2}}+\frac{v_{\mathrm{gas}}}{1+\mathrm{St}_{i}^{2}}$,

where $v_{\text {gas }}$ is the local gas velocity (driven by gas viscous evolution), and $v_{\eta}$ is the maximum velocity of the radial drift driven by the local pressure gradient $\partial_{\mathrm{r}} P_{\text {gas }}$. We calculate the $v_{\eta}$ as

$v_{\eta}=\frac{\partial_{r} P_{\mathrm{gas}}}{2 \rho_{\mathrm{gas}} \Omega_{\mathrm{K}}}$,

where $\rho_{\text {gas }}=\Sigma_{\text {gas }} /\left(\sqrt{2 \pi} H_{\text {gas }}\right)$ is the midplane density of gas.

We took into account both the Epstein and the Stokes drag regimes when calculating the Stokes number $\mathrm{St}_{i}$. We did not include the back-reaction from dust to gas in this work. Further details about the dust evolution code can be found in Birnstiel et al. (2010) and in a forthcoming paper by Stammler \& Birnstiel (in prep.).

\subsection{Planetary growth by pebble accretion}

We considered the growth of planetary embryos with starting masses of $0.001 M_{\oplus}$ (approximately 0.1 Moon mass, or 7 masses of the largest asteroid, Ceres). We modeled the growth of embryos at various radial locations and did not consider their migration or planet-disk interaction. We did not subtract the dust accreted by an embryo from the dust evolution code, which means that we assume that each model corresponds to a single planet. We considered various possibilities for the point at which we would insert the embryos into the simulation $t_{0}$ : either we let them start to grow from the beginning of the simulation, or after
$10^{5}$ and $10^{6} \mathrm{yr}$. We assumed that the embryos are on circular orbits with zero inclination.

To calculate the pebble accretion efficiency, we used the method provided by Liu \& Ormel (2018) and Ormel \& Liu (2018), who used three-body calculations to obtain a general recipe for the pebble accretion efficiency of a single planet $^{1}$. In Fig. 2, we show the example of pebble accretion efficiencies obtained using this recipe for a range of planetary embryo masses and sizes at 5 and 30 au in the initial condition of the compact disk with the turbulence parameter $\alpha=10^{-4}$. As can be seen in this figure, the recipe takes into account both the 2D regime, where the embryo is large enough to accrete from the complete layer of pebbles, and the 3D regime, where the small embryo only has access to a fraction of the pebble layer. The transition from a $3 \mathrm{D}$ to $2 \mathrm{D}$ regime is marked with the dasheddotted line. The gray, dashed line shows the transition between the headwind and shear regimes. The headwind regime, also called the Bondi regime, is valid for small embryos or small pebbles, when the pebbles are accreted only from the embryo proximity, and the relative velocity between the pebbles and the embryo is determined by the gas flow. Larger embryos enter the shear regime, also called the Hill regime, when the pebbles are accreted from a much larger area, and their approach velocity is determined by the Keplerian shear. The transition between the headwind and shear regimes does not impact the pebble accretion efficiency in the $3 \mathrm{D}$ regime, while in the $2 \mathrm{D}$ regime it slightly changes the dependence of $\varepsilon_{\mathrm{PA}}$ on the Stokes number. This effect becomes more important in the outer regions of the disk, when the transition between headwind and shear regime shifts to higher embryo masses. As explained in Ormel (2017), in the 3D regime the pebble accretion efficiency increases with the Stokes number, because dust settling is more efficient and the embryo accretes from a denser midplane layer; while in the 2D regime, this effect is canceled by the increasing drift speed of pebbles and the decreasing embryo-pebble interaction time.

\footnotetext{
1 The Python implementation of this algorithm is publicly available at https://staff. fnwi .uva.nl/c.w.ormel/software/epsilon. tar.gz
} 
The dotted line in Fig. 2 shows the pebble accretion onset, which is the minimum embryo mass for which the aerodynamic deflection becomes important (Visser \& Ormel 2016). For reference, the typical planetesimal size formed in the streaming instability models is about $100 \mathrm{~km}$ (Schäfer et al. 2017; Li et al. 2019a; Klahr \& Schreiber 2020). Interestingly, these smallest embryos could only grow by accretion of small pebbles and with an extremely low efficiency. The pebble accretion onset shifts toward even larger embryo masses in the outer parts of the disk.

Figure 2 shows that the pebble accretion efficiency is mostly dependent on the embryo mass and not on the pebble size, as long as the Stokes number stays between $10^{-3}$ and 1 , in the typical "pebble-size" regime. A relatively large embryo with a significant fraction of Earth mass is able to grow quickly by accreting a considerable fraction of the pebble flux. It is worth noting that multiple small embryos could grow at the same time, collectively capturing a similar fraction of pebbles as a single large embryo (Bitsch et al. 2019b). The growth rate of the planetary embryo depends both on the pebble accretion efficiency and the pebble flux. In this paper, we compute pebble fluxes consistently with the pebble size resulting from our full dust coagulation model.

Our pebble accretion algorithm works as follows. At the location of the planetary embryo, $a_{\mathrm{e}}$, we calculate the Stokes number of the dust particles from every mass bin, $\mathrm{St}_{i}$. Then, we calculate the pebble accretion efficiency $\varepsilon_{\mathrm{PA}}\left(m_{\mathrm{e}}, a_{\mathrm{e}}, \mathrm{St}_{i}\right)$ for the current embryo mass, $m_{\mathrm{e}}$, and multiply it by the flux of solids corresponding to each bin of the mass grid, $\dot{m}_{\mathrm{p}}\left(a_{\mathrm{e}}, \mathrm{St}_{i}\right)$, obtained in the dust evolution code. In the next step, we sum the contributions from each dust mass bin to obtain the embryo growth rate:

$\dot{m}_{\mathrm{e}}=\sum_{i} \varepsilon_{\mathrm{PA}}\left(m_{\mathrm{e}}, a_{\mathrm{e}}, \mathrm{St}_{i}\right) \cdot \dot{m}_{\mathrm{p}}\left(a_{\mathrm{e}}, \mathrm{St}_{i}\right)$.

The growth stops when the embryo reaches the pebble isolation mass. Following Lambrechts et al. (2014), we calculate the pebble isolation mass as

$M_{\text {iso }}=20 M_{\oplus} \cdot\left(\frac{H_{\mathrm{gas}} / r}{0.05}\right)^{3}$,

where $H_{\text {gas }} / r$ is the disk's aspect ratio. It is worth noting that Ataiee et al. (2018) and Bitsch et al. (2018b) reported that the pebble isolation mass may be increased in turbulent disks, while Zormpas et al. (2020) found that the pebble isolation mass is decreased when a realistic equation of state and radiative cooling are taken into account.

\section{Results}

We performed seven different models, varying the fragmentation threshold velocity, disk size, and turbulence level. In each of the models, we considered the growth of planetary embryos by pebble accretion at $1,5,10,20,30,40$, and 50 au. However, in none of the models did an embryo located outside of 20 au reach the pebble isolation mass.

\subsection{Fragmentation threshold}

We first focused on the impact of the fragmentation threshold value on planetary growth. Figure 3 presents results of four runs, all performed with the compact disk with the turbulence strength $\alpha=10^{-4}$ and different fragmentation threshold speeds: $v_{\mathrm{f}}=1 \mathrm{~m} \mathrm{~s}^{-1}, v_{\mathrm{f}}=2 \mathrm{~m} \mathrm{~s}^{-1}, v_{\mathrm{f}}=10 \mathrm{~m} \mathrm{~s}^{-1}$, and $v_{\mathrm{f}}=\infty$ (corresponding to no fragmentation). The left panel of Fig. 3 presents the growth of planetary embryos at 1, 5, 10, and 20 au. At each location, we tested different embryo introduction times, $t_{0}$ : at the beginning of the simulation $\left(t_{0}=0\right)$, after $10^{5} \mathrm{yr}$, and after $10^{6} \mathrm{yr}$ of dust evolution.

The runs with $v_{\mathrm{f}}=10 \mathrm{~m} \mathrm{~s}^{-1}$ and no fragmentation produced very similar results except for the inner region of the disk ( $r \leq$ $5 \mathrm{au}$ ). Interestingly, in this inner region of the disk, the growth of embryos introduced at $t_{0}=0$ and $t_{0}=10^{5} \mathrm{yr}$ proceeds faster in the runs with fragmentation than in the run without fragmentation. In the run without fragmentation, the embryo located at 1 au does not reach its pebble isolation mass, irrespective on the introduction time; while in the run with $v_{\mathrm{f}}=10 \mathrm{~m} \mathrm{~s}^{-1}$, the innermost embryo reaches the isolation mass after only tens of thousands of years. The reason for the slow growth of the close-in embryos in the model without fragmentation is that pebbles break through the drift barrier and continue to grow quickly over the Stokes number of unity, in the Stokes drag regime (this process has been previously described in the literature; see e.g., Brauer et al. 2008a; Birnstiel et al. 2010; Okuzumi et al. 2012; Laibe 2014; Drążkowska et al. 2014).

The breakthrough only happens in the inner part of the disk, as the outer part is still dominated by the radial drift barrier. Figure 4 presents the average Stokes numbers and grain sizes as a function of radial distance at $t=2 \times 10^{5} \mathrm{yr}$ of evolution in the four models with different fragmentation thresholds. It is worth noting that the flux-averaged dust size (and Stokes number) is typically slightly larger than the mass-averaged value, except for the grains that break through the $\mathrm{St}=1$ barrier. This is because the radial drift velocity increases up to $\mathrm{St}=1$ and then decreases again (see Eq. (3)). The grain size of $\sim 100 \mathrm{~m}$ reached in the inner region in the run without fragmentation is an effect of the upper limit of the size grid used in the dust coagulation calculation rather than a physical value. The large solids do not drift efficiently, which manifests as the low pebble flux at 1 au in the right panel of Fig. 3. Since the grain growth is limited to the upper boundary of the size grid, those results should be treated as approximate, as the pebble flux would drop even more if the growth could proceed without a limit.

Except for reducing the pebble flux, breaking through the St $>1$ barrier has another negative impact on the planetary growth rate. The efficiency of pebble accretion significantly drops for such large dust aggregates (see Fig. 2), as technically they no longer count as pebbles. Thus, even though the total mass of solids passing around the close-in embryo over the disk's lifetime is essentially the same, in the run without fragmentation the planet does not reach the pebble isolation mass.

This result shows that some level of dust fragmentation may be beneficial to planetary growth via pebble accretion. Thus, we performed additional runs, lowering the fragmentation speed even more, to $v_{\mathrm{f}}=2$ and $v_{\mathrm{f}}=1 \mathrm{~m} \mathrm{~s}^{-1}$, the latter value being roughly consistent with the conclusions from laboratory work (Güttler et al. 2010). As is visible in the right panel of Fig. 3, decreasing the fragmentation speed leads to pebble flux reduction. In the runs with the low fragmentation speed, grains remain smaller in size (see Fig. 4), and drift is slower, leading to lower but long-lasting mass flux, as the depletion of small aggregates takes longer. This effect is particularly important if the planetary embryo takes a long time to form. In the left panel of Fig. 3, one can see that if embryos are introduced at 1 Myr of dust evolution, they grow the most in the run with the lowest fragmentation threshold, $v_{\mathrm{f}}=1 \mathrm{~m} \mathrm{~s}^{-1}$.

Interestingly, for embryos introduced at $t_{0}=10^{5} \mathrm{yr}$, the $v_{\mathrm{f}}=2 \mathrm{~m} \mathrm{~s}^{-1}$ is the most favorable value in terms of planetary growth. This is because, while the flux in the runs with the higher 

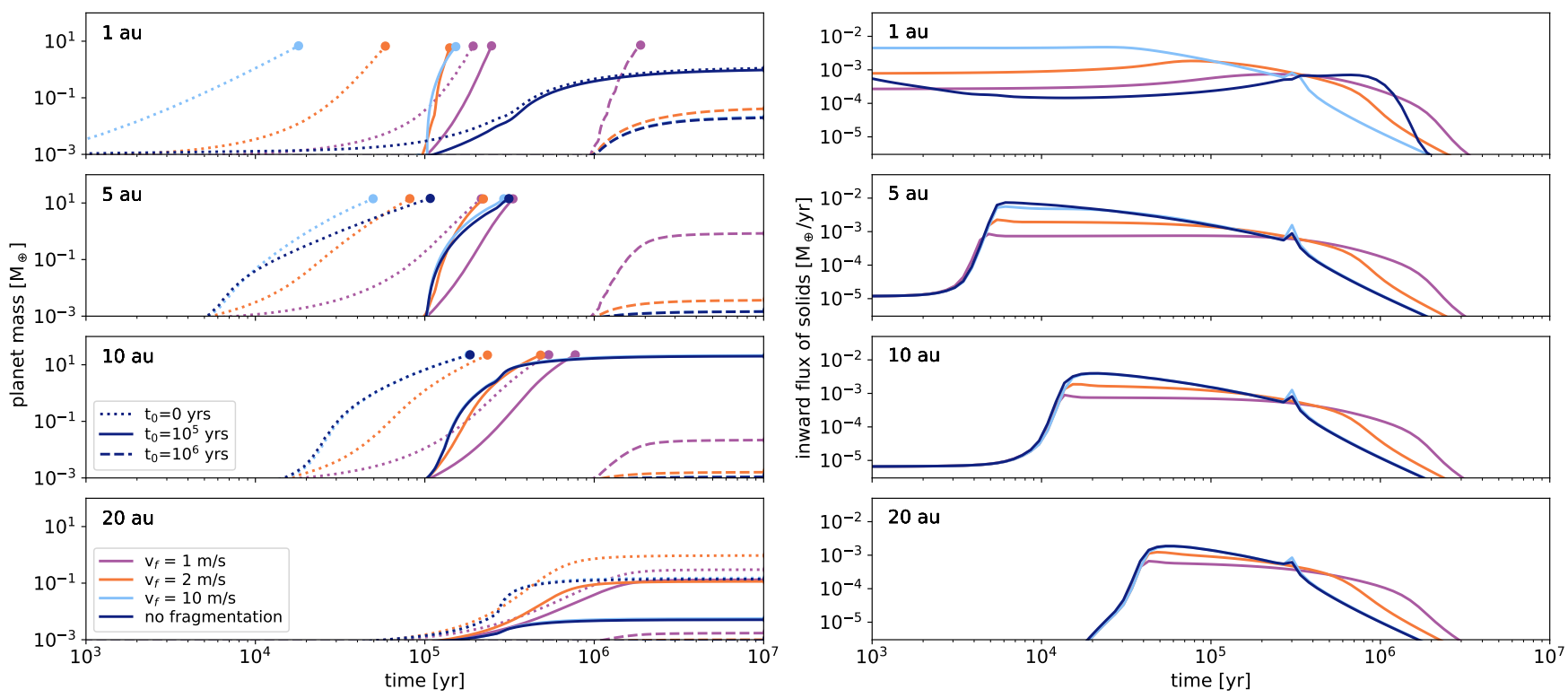

Fig. 3. Left: planetary embryo growth via pebble accretion in the compact protoplanetary disk in models with $\alpha=10^{-4}$ and different fragmentation threshold speeds and one not including dust fragmentation at all. In all cases where the line representing the $v_{\mathrm{f}}=10 \mathrm{~m} \mathrm{~s}^{-1}$ run is not visible, it is identical to the "no fragmentation" run. Lines ending with a circle represent growth tracks that reached the pebble isolation mass. Right: inward flux of solids (all dust sizes integrated) at different locations in the same models.

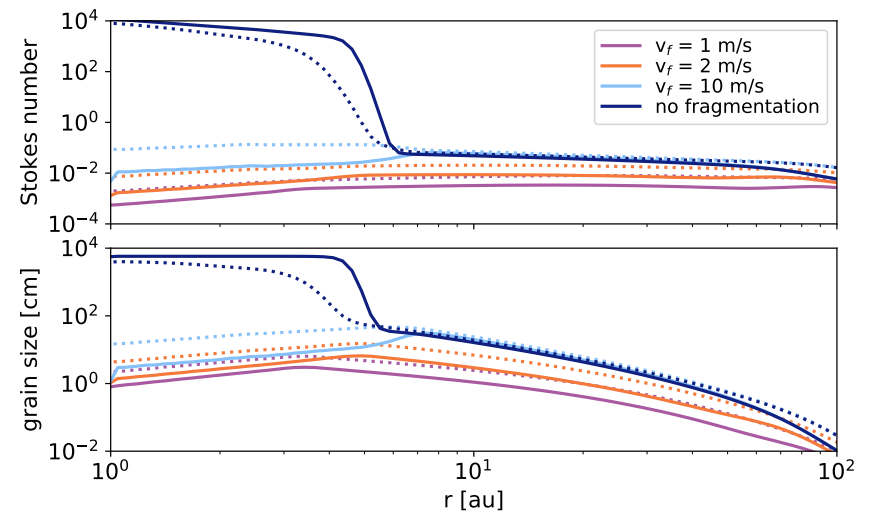

Fig. 4. Mass-averaged (solid lines) and flux-averaged (dotted lines) Stokes number (upper panel) and grain size (lower panel) after $2 \times$ $10^{5} \mathrm{yr}$ of evolution in the models of a compact protoplanetary disk with $\alpha=10^{-4}$ and varying fragmentation threshold velocity $v_{\mathrm{f}}$.

fragmentation threshold is already decreasing at that point, the flux in the $v_{\mathrm{f}}=2 \mathrm{~m} \mathrm{~s}^{-1}$ run stays high for another couple of hundred thousand years (see the right panel of Fig. 3). At the same time, the flux is factor of two higher in the $v_{\mathrm{f}}=2 \mathrm{~m} \mathrm{~s}^{-1}$ run than in the $v_{\mathrm{f}}=1 \mathrm{~m} \mathrm{~s}^{-1}$ case.

\subsection{Disk size}

In the results presented in Sect. 3.1, the pebble flux lasts for at most 3 Myrs. However, millimeter- and centimeter-sized pebbles are observed in protoplanetary disks older than a few Myr (Wilner et al. 2005; Ricci et al. 2010). Johansen et al. (2019, their Appendix A) noticed this problem and speculated that limited dust growth in the outer part of the disk may be the solution for retaining pebbles over long timescales. They also showed that large initial disk size facilitates long-lasting pebble flux, a solution often used in pebble accretion models (Lambrechts \& Johansen 2014; Sato et al. 2016; Bitsch et al. 2018a). While large disks are bright and thus relatively easy to image at high angular resolution (Andrews et al. 2018), there is evidence that most of the disks may, in fact, be small (Ansdell et al. 2018; Long et al. 2019; Maury et al. 2019; Tobin et al. 2020; Trapman et al. 2020). In this section, we study the impact of disk size on the pebble flux and resulting planetary growth.

Figure 5 presents the results of two models where the same initial mass was distributed, either in a compact disk with the critical radius $r_{\mathrm{c}}=30 \mathrm{au}$, or in a much larger disk with $r_{\mathrm{c}}=300 \mathrm{au}$. The other simulation parameters are the same: the fragmentation speed is $v_{\mathrm{f}}=10 \mathrm{~m} \mathrm{~s}^{-1}$ and the turbulence strength is $\alpha=10^{-4}$.

The compact disk produces a very high pebble flux (well over $10^{-3} M_{\oplus} \mathrm{yr}^{-1}$ ), which, however, only lasts for about $3 \times 10^{5} \mathrm{yr}$ and declines over the next couple of hundred thousand years (see the right panel of Fig. 5). With such a high pebble flux, the embryos introduced at the beginning of the simulation take less than one hundred thousand years to reach their isolation mass. The embryo placed at 1 au reaches its isolation mass in little over ten thousand years, which is the fastest growth we observe in any of our runs.

Pebble flux obtained in the large disk is almost one order of magnitude lower than that in the compact disk, but it lasts proportionally longer. Thus, even embryos introduced late during the simulation, at $t_{0}=10^{6} \mathrm{yr}$, are able to obtain their isolation mass, at least inside $10 \mathrm{au}$. Therefore, extending the initial size of the disk gives similar results to lowering the fragmentation speed, as we discussed in the previous section. There is, however, one important difference, which manifests itself in the planetary growth results at and outside 10 au. In the compact disk, a significant fraction of the mass is initially located inside $10 \mathrm{au}$. The mass reservoir available for wide-orbit planets is significantly higher in the large disk than in the compact disk. Hence, for example, the embryo introduced at $t_{0}=10^{5} \mathrm{yr}$ at 10 au reaches its isolation mass in the large disk but not in the compact disk.

With the large mass reservoir available even at wide orbital distances, it may be surprising that no embryo reaches its isolation mass outside 10 au in the large disk. This is because the pebble accretion efficiency for a constant embryo size significantly 

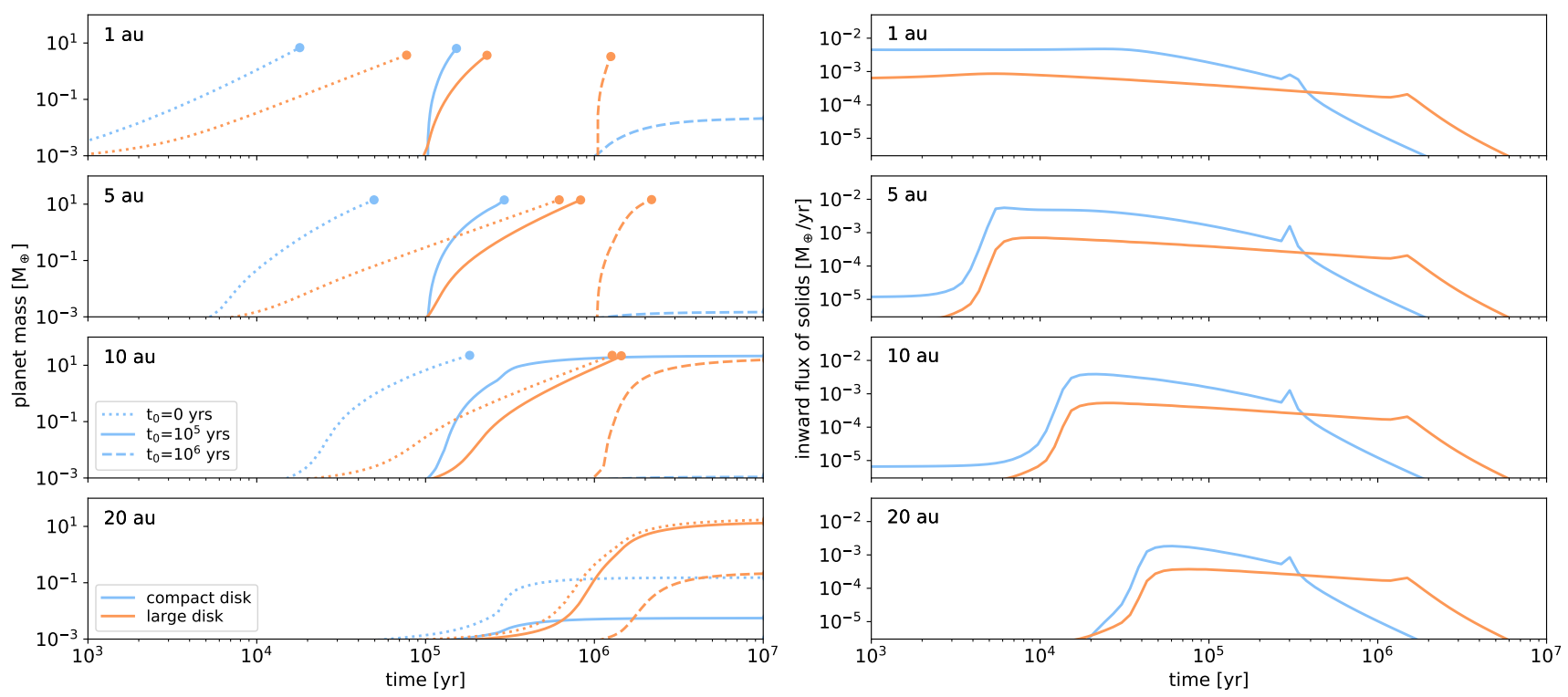

Fig. 5. Left: Planetary embryo growth via pebble accretion in models with a different characteristic radius, $\alpha=10^{-4}$, and the fragmentation threshold of $v_{\mathrm{f}}=10 \mathrm{~m} \mathrm{~s}^{-1}$. Lines ending with a circle represent growth tracks that reached the pebble isolation mass. Right: inward flux of solids (all dust sizes integrated) at different locations in the same models.

decreases with radial distance (see Fig. 2). In this paper, we consider the initial embryo mass of $0.001 M_{\oplus}$ irrespective of its location (and starting time). More massive embryos could probably still reach pebble isolation mass in the outer parts of the disk.

\subsection{Turbulence}

The turbulence level in the protoplanetary disk is a fundamental unknown factor. Observational constraints on the disk dispersal timescales suggest turbulence levels of $\alpha \approx 10^{-2}$ if the dispersal were propelled solely by accretion driven by the turbulent transportation of angular momentum (Hernández et al. 2007). However, more recent disk models suggest that the turbulence level may vary within the disk and that large regions of the disk, particularly near to the midplane, may have low levels of turbulence (Lesur et al. 2014; Bai 2016). This would be consistent with the disk images obtained in millimeter wavelengths, where the pebbles appear to be well-settled (Pinte et al. 2016; Villenave et al. 2020), and also consistent with the low upper limits set by measurements of turbulent line broadening (Flaherty et al. 2015, 2020; Teague et al. 2016, 2018). Given the uncertainties and difficulties in constraining the turbulence level observationally, in this section, we consider the turbulence strength $\alpha$ as a free parameter.

Figure 6 shows results of three runs performed on the backdrop of the compact disk model with different levels of turbulence. We keep the fragmentation speed at $v_{\mathrm{f}}=1 \mathrm{~m} \mathrm{~s}^{-1}$ in all the runs. As is visible in the left panel of Fig. 6, low turbulence promotes planetary growth irrespective of the distance to the star and the starting time of the embryo. There are multiple reasons for this. First of all, a lower turbulence level leads to the better settling of the pebbles and higher pebble accretion efficiency (Ormel 2017). Lower turbulence speed promotes growth to larger pebble sizes, which increases the pebble flux, although this effect stops when the fragmentation starts to be dominated by the differential radial drift rather than turbulence (this happens for $\alpha \lesssim 10^{-4}$; see the right panels of Fig. 6).

It is worth noting that in case of the highest turbulence parameter value that we considered, $\alpha=10^{-3}$, no embryo reaches the pebble isolation mass. If the turbulence is strong, with $\alpha \geq$ $10^{-3}$, the fragmentation threshold velocity needs to be much higher than $v_{\mathrm{f}}=1 \mathrm{~m} \mathrm{~s}^{-1}$ (which we considered in models presented in Fig. 6) to allow planetary embryos to benefit from pebble accretion.

\section{Simple prediction of the pebble flux}

As we show above, planetary growth by pebble accretion is very sensitive to disk parameters and dust evolution details. The pebble flux depends on the initial mass budget, disk size, turbulence level, and fragmentation threshold speed. The pebble accretion efficiency is sensitive to pebble size and settling. The DustPy simulations described above are relatively expensive, typically taking several days of computations. In this paper, we propose a simple and efficient method that predicts the size and flux of pebbles for an arbitrary protoplanetary disk without substructure, the pebble predictor ${ }^{2}$.

The general mechanics of pebble predictor is closely related to two-pop-py presented by Birnstiel et al. (2012), although a full time integration of the disk is not performed. The pebble predictor uses the initial state of the gas and dust disk surface densities, $\Sigma_{\text {gas }, 0}$ and $\Sigma_{\text {dust }, 0}$, temperature, $T$, turbulence strength, $\alpha$, and fragmentation threshold velocity, $v_{\mathrm{f}}$, to predict the flux-averaged Stokes number and total flux of pebbles at every location within the disk and at any time point.

It is assumed that all the dust grains are initially in the form of micron-sized monomers and that they grow in collisions driven by turbulence, which leads to the growth timescale of

$\tau_{\text {growth }}=\frac{\Sigma_{\text {gas }, 0}}{\Sigma_{\text {dust }, 0}} \cdot \Omega_{\mathrm{K}}^{-1} \cdot\left(\frac{\alpha}{10^{-4}}\right)^{-1 / 3} \cdot\left(\frac{r}{\mathrm{au}}\right)^{1 / 3}$,

where $\Omega_{\mathrm{K}}$ is the Keplerian frequency and $r$ is the radial distance to the central star. The growth proceeds as an exponential

2 pebble predictor is publicly available at https://github.com/astrojoanna/pebble-predictor. Version 1.0 of the script used in this paper is permanently available at https://doi.org/10.5281/zenodo. 4383153 

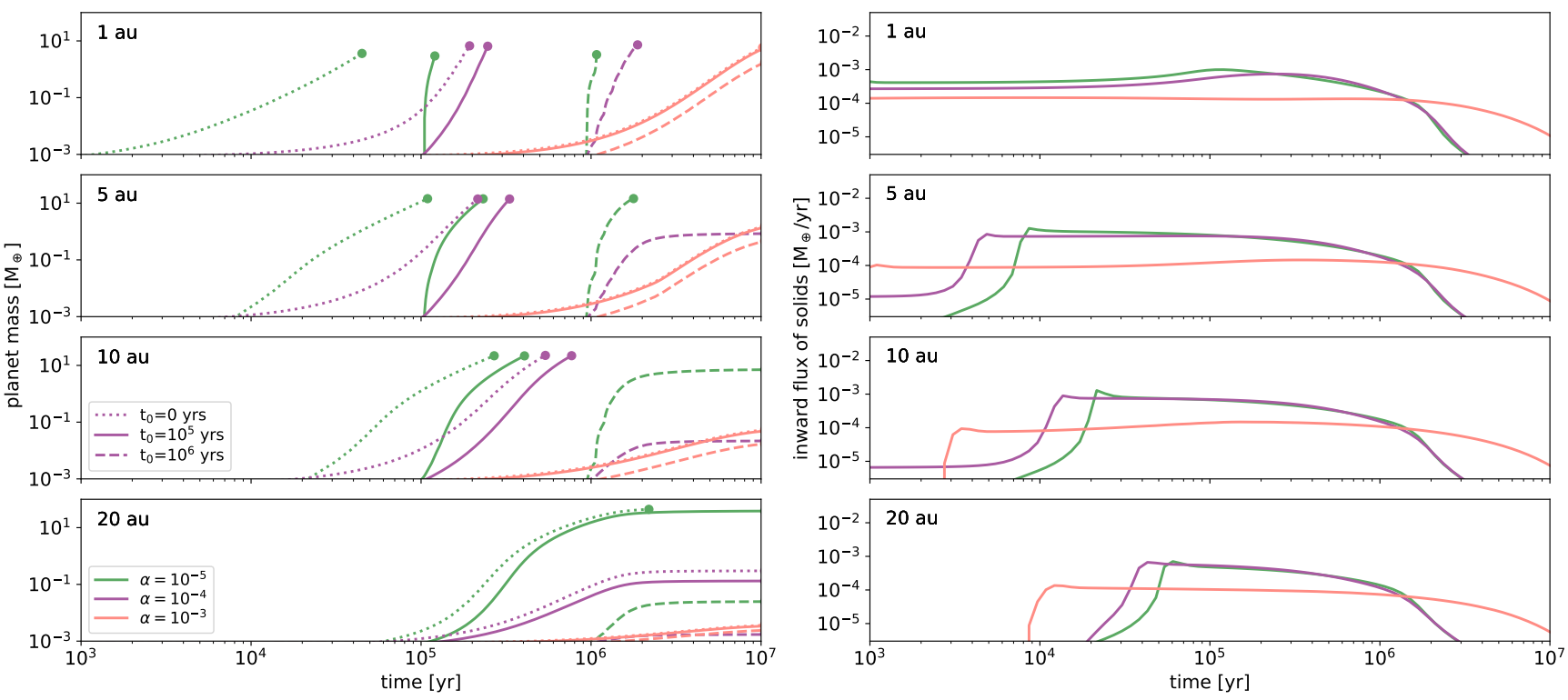

Fig. 6. Left: planetary embryo growth via pebble accretion in the compact disk models with different turbulence strength parameters, $\alpha$, and the fragmentation threshold of $v_{\mathrm{f}}=1 \mathrm{~m} \mathrm{~s}^{-1}$. Lines ending with a circle represent growth tracks that reached the pebble isolation mass. Right: inward flux of solids (all dust sizes integrated) at different locations in the same models.

function of time, $t$ :

$\mathrm{St}_{\text {ini }}=\mathrm{St}_{0} \cdot \exp \left(\frac{t}{\tau_{\text {growth }}}\right)$,

where $\mathrm{St}_{0}$ is the Stokes number corresponding to the micronsized monomers, until one of the growth barriers is encountered: fragmentation or radial drift. Fragmentation may be driven by turbulence or the differential radial drift. The maximum Stokes number of dust aggregates achievable with respect to the turbulent fragmentation is

$\mathrm{St}_{\mathrm{f}}=f_{\mathrm{f}} \frac{v_{\mathrm{f}}^{2}}{3 \alpha c_{\mathrm{s}}^{2}}$,

where $f_{\mathrm{f}}$ is a parameter, $v_{\mathrm{f}}$ is the fragmentation threshold velocity, $\alpha$ is the turbulence strength parameter, and $c_{\mathrm{s}}$ is the sound speed. If the radial drift speeds dominate over the turbulent speeds, the maximum Stokes number is

$\mathrm{St}_{\mathrm{df}}=f_{\mathrm{f}} \frac{v_{\mathrm{f}}}{2 v_{\eta}}$,

where $v_{\eta}$ is the maximum drift speed (see Eq. (4)). We adopted $f_{\mathrm{f}}=0.37$.

We assume that the growth needs to be $f_{\mathrm{d} / \mathrm{g}}=30$ times faster than drift in order for the grains not to be impacted by the radial drift (see Okuzumi et al. 2012, their Sect. 4). We calculate the size of grains that are impacted by radial drift by checking the condition $\tau_{\text {drift }}=f_{\mathrm{d} / \mathrm{g}} \cdot \tau_{\text {growth }}$, which leads to

$\mathrm{St}_{\mathrm{drift}}=\frac{1}{f_{\mathrm{d} / \mathrm{g}} \eta} \cdot \frac{\Sigma_{\mathrm{dust}}}{\sum_{\mathrm{gas}}}$,

where $\eta=v_{\eta} / v_{\mathrm{K}}$ is the pressure gradient parameter. The resulting representative Stokes number, St, in every radial bin is then chosen as a minimum of $\mathrm{St}_{\text {ini }}, \mathrm{St}_{\mathrm{f}}, \mathrm{St}_{\mathrm{df}}$, and $\mathrm{St}_{\text {drift }}$; however, it is not allowed to fall below its initial value, $\mathrm{St}_{0}$.

We used the first part of Eq. (3) to calculate the radial drift speed, $v_{\mathrm{r}}$, corresponding to the representative Stokes number, and we were able to calculate the pebble flux as

$\dot{m}_{\mathrm{p}}=2 \pi r v_{\mathrm{r}} \Sigma_{\text {dust }}$, where $\Sigma_{\text {dust }}$ would be the surface density of dust at a given time. This equation is, however, only correct if the grains grow faster than they drift. In such a case, there are always "enough" large grains, as the drift depletes them at a slower rate than the growth can replenish them. In the opposite case, the pebble flux needs to be limited; therefore, we take into account the fact that the supply of large grains can only be replenished at the growth timescale $\tau_{\text {growth. }}$ Thus, we limited the inward drift velocity, $v_{\mathrm{r}}$, to

$v_{\mathrm{r}}=\min \left(\frac{2 v_{\eta} \mathrm{St}}{1+\mathrm{St}^{2}}, \frac{r}{f_{\mathrm{d} / \mathrm{g}} \tau_{\text {growth }}}\right)$,

which gives us a good estimate of the flux, both in the fragmentation-dominated and drift-dominated regions of the disk.

As mentioned above, pebble predictor does not perform a full time integration of the disk. We neglected the evolution of the gas disk, and we approximated the dust surface density evolution by keeping track of the mass budget. Using the initial $\Sigma_{\text {dust, } 0}$ profile supplied as an input, we calculated the initial mass, $M_{\text {out, } 0 \text {, outside every radial grid point. Using a time grid supplied }}$ as an input, pebble predictor calculates how much mass is left outside every radial grid point at every time point, $i$, using the values obtained in the previous time step:

$M_{\text {out }, \mathrm{i}}=M_{\text {out }, \mathrm{i}-1}-\dot{m}_{\mathrm{p}, i-1} \cdot\left(t_{i}-t_{i-1}\right)$.

The dust surface density used in Eqs. (11-12) is then approximated as $\Sigma_{\text {dust,i }}=\Sigma_{\text {dust }, 0} \cdot M_{\text {out, },} / M_{\text {out }, 0}$. Since the time steps supplied in the input time grid do not necessarily fulfill the CFL condition, this routine cannot be treated as a rigorous integration. Its goal is to correct the pebble flux for the remaining mass budget rather than recovering the actual surface density evolution.

Figure 7 shows the comparison of the pebble predictor and DustPy results for various setups varying the disk size, fragmentation speed, and turbulence level. The representative Stokes number from pebble predictor is benchmarked against the flux-averaged Stokes number from the full coagulation calculation. In the top-right panel of Fig. 7, we additionally show the 

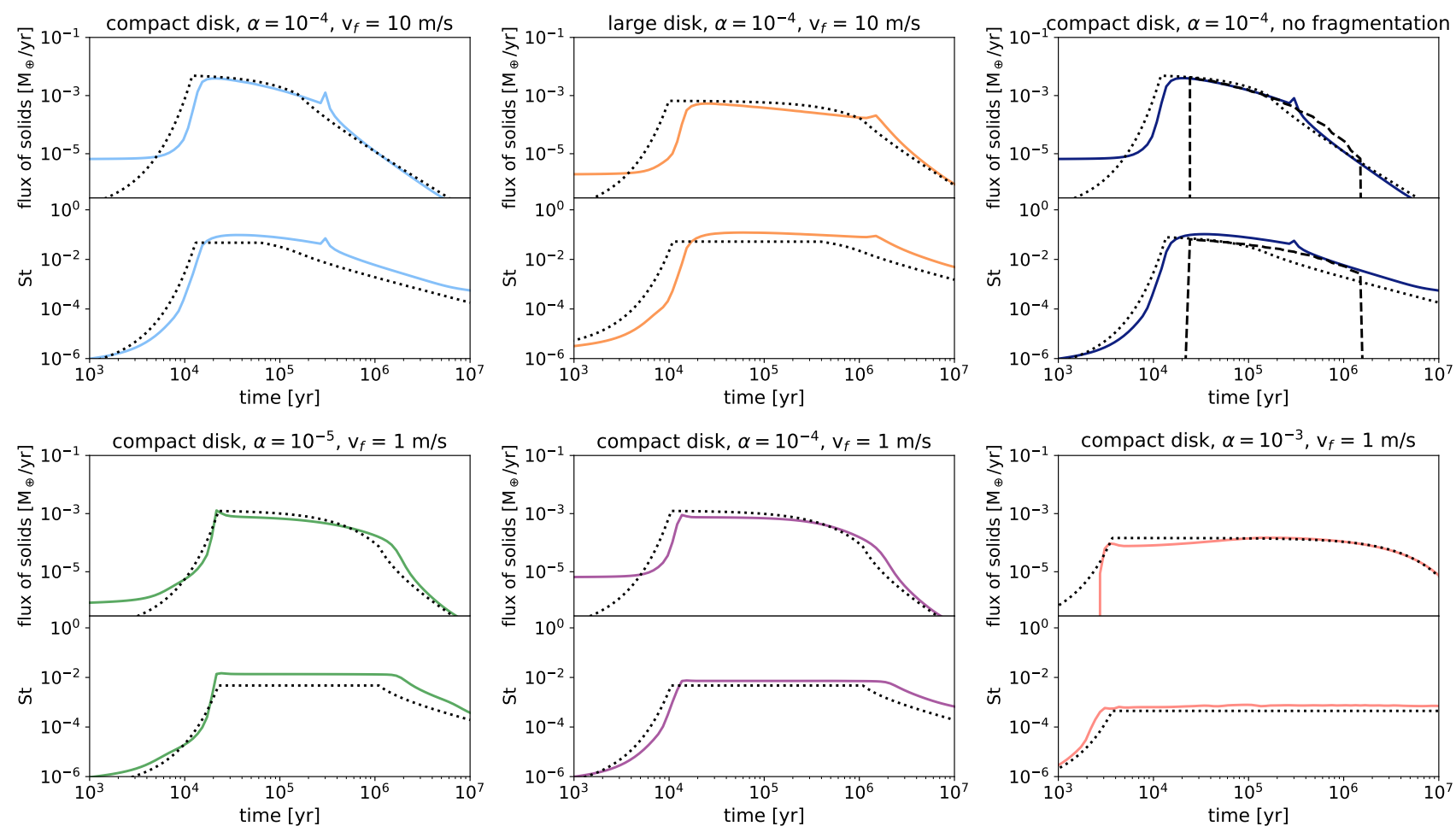

Fig. 7. Comparison of pebble flux and Stokes number predictions and simulation results for different models, as indicated by the title of each subplot at $10 \mathrm{au}$. The solid lines show the DustPy results, the dotted lines come from the pebble predictor. The dashed line in the top right panel corresponds to the model proposed by Lambrechts \& Johansen (2014), which can only be used if the fragmentation is not included.

flux and the Stokes number prediction from the analytical model proposed by Lambrechts \& Johansen (2014). In this model, it is assumed that the pebble flux is set by the initial dust surface density at the pebble formation front, which spreads radially with time. The general outcome of this model fits our results well, although the growth timescale is slightly underestimated, and the pebble flux starts later than in the numerical results. The most significant difference is revealed at later times, as the pebble flux in the Lambrechts \& Johansen (2014) model abruptly stops when the pebble formation front reaches the outer edge of the disk. In reality, however, viscous spreading of the disk and dust diffusion modify the shape of the outer disk edge before the grains there reach pebble sizes. Moreover, not all the pebbles at a given location grow and start drifting at once, which also leads to the extension of the duration of the pebble flux. Finding an analytical prediction that would encompass all the relevant processes is not feasible. Thus, the pebble predictor is a semianalytic model relying on some empirical scaling.

The main limitations of pebble predictor, causing some discrepancies between its predictions and the full simulation results are summarized here. The pebble predictor assumes that the growth is driven by turbulence (only one of the turbulent regimes calculated by Ormel \& Cuzzi 2007, to be precise). This assumption breaks in the case of low turbulence, when the collisions are mostly driven by differential drift. For very small particles, the turbulent speeds fall into a different regime (see also the discussion in Powell et al. 2019, their Appendix B), which leads to the need to modify the growth timescale prescription with the empirical dependences on turbulent strength, $\alpha$, and the radial distance in Eq. (7). Only the Epstein drag regime is considered, which leads to error when estimating both the size and pebble flux where the growth enters the Stokes regime, so in the inner parts of disks in runs where dust fragmentation is not considered (see Sect. 3.1).
Gas disk evolution is not included, leading to inaccuracies particularly in the outer regions of the disk, where disk spreading slows down the flow of pebbles. Similarly, dust advection with the gas flow is not considered in pebble predictor, which may lead to issues in estimating both the flux and pebble size correctly in runs where a significant fraction of solids are coupled to the gas; for example, when the turbulence level is high and the fragmentation threshold is low. What is more, diffusion is not considered, which is particularly important at the outer edge of the disk, where the dust-to-gas ratio gradient is strong (Birnstiel \& Andrews 2014). This leads to the characteristic peak in the dust flux visible in the runs involving the compact disk and a high fragmentation threshold, a feature which is not reproduced by the pebble predictor.

Because the dust advection with gas flow and diffusion are not included, pebble predictor should not be applied to disks with a substructure (see Sect. 5.5). A full model, such as DustPy or a more advanced simplified method, such as two-pop-py developed by Birnstiel et al. (2012), may be a better choice in such cases.

Considering all the limitations, the pebble predictor does surprisingly well at predicting the dependences of pebble flux and size on the fragmentation speed, disk size, and turbulence level, as demonstrated in Fig. 7.

\section{Discussion}

\subsection{Limitations of the (full) model}

In this paper, we coupled dust evolution calculated with the DustPy code and embryo growth by pebble accretion. Our models have certain limitations, and some of them may impact the planetary growth results discussed in this paper. 
First of all, we do not consider that dust grains are composed of various materials and that the evaporation of volatile components could cause a decrease in the pebble flux in the inner regions of the disk. We assume that all the dust aggregates are compact, with constant internal density, and neglect the effects of porosity.

The structure of the gas disk is independent of the evolution of solids. This is valid as long as the solid-to-gas ratio stays low, which is true in almost all of the models except for the one without fragmentation, when the grains break through the $\mathrm{St}=1$ barrier and pile up in the inner part of the disk.

Our models correspond to a one-planet-per-disk approach, as we do not decrease the pebble reservoir by the amount accreted by each planet. Similarly, we do not include perturbations to the disk structure caused by the growing planets. Such multiplanetary effects could be important, as many embryos would grow from the same pebble reservoir, and the outermost embryo would block the pebble flux when it reaches its pebble isolation mass.

In this paper, we assume that the disk is fully turbulent, with a constant $\alpha$ value. Modern disk models predict that large parts of the disk may be free from turbulence and that the accretion is primarily driven by magnetized disk winds (Lesur et al. 2014; Bai 2017). In such disks, the viscous heating would not be relevant, leading to lower temperatures in the inner part of the disk than we assume in our models (Mori et al. 2019). Lower temperatures typically promote pebble accretion, as the grains grow to larger sizes and are more settled. On the other hand, the pebble isolation mass is lower for colder disks. Similarly, we do not include the gas dispersal caused by effects other than viscous accretion. Magnetic and photoevaporative winds could completely change the disk's structure, particularly in the later phases of its evolution, which would impact the pebble fluxes and probably abruptly stop planetary growth by pebble accretion.

\subsection{Planet migration}

Perhaps the most significant limitation of our models is that we do not include planetary migration. Interaction between a sufficiently massive planetary core and the gas disk leads to radial migration (Ward 1997). A typical timescale of the inward migration for an Earth-mass planet at 1 au is $10^{5}$ yr (Ogihara et al. 2015), which is comparable to the timescale of planetary growth by pebble accretion.

Many authors have studied the interplay between the planetary migration and planetary growth in a pebble accretion scenario. Bitsch et al. (2015) showed that planets mostly migrate in the type I regime while accreting pebbles, but the migration direction may be both inward and outward, depending on their radial location. Planets that reach masses allowing for gas accretion typically outgrow the outward migration region and significantly change their radial location by tens of au. Nevertheless, planetary growth by pebble accretion is generally fast enough for planets to reach the pebble isolation mass before falling onto the central star due to migration (Bitsch \& Johansen 2016; Ndugu et al. 2018; Johansen et al. 2019).

A connection between planet migration and pebble accretion including the self-consistent dust evolution model will be a subject of our future work.

\subsection{Validity of the single dust size approach in pebble accretion models}

In the models presented in this paper, we used a dust evolution model that includes the distribution of dust sizes. For every size bin, we used the solid flux calculated by the DustPy code and multiplied it by the pebble accretion efficiency factor, $\varepsilon_{\mathrm{PA}}$, corresponding to this pebble size, to obtain the pebble accretion rate. We summed the contributions from each size bin to calculate planet accretion rate (see Eq. (5)) ${ }^{3}$. However, since this type of model is computationally expensive, many authors employ simplified models of dust evolution. For example, the two-population model for dust evolution proposed by Birnstiel et al. (2012) is commonly used to compute dust evolution in protoplanetary disks, as it allows us to recover the dust density evolution at a relatively low computational cost, without solving the full coagulation equation (see e.g., Drążkowska et al. 2016; Cridland et al. 2017; Tamfal et al. 2018; Charnoz et al. 2019; Gárate et al. 2020).

In this paper, we propose an even simpler way of predicting pebble size and flux based on one representative grain size at every distance, the pebble predictor (see Sect. 4). One would be justified in asking whether these simple models can be reliably used to calculate planetary growth via pebble accretion even though they do not calculate the full size distribution. We tested this by using the results of pebble predictor to calculate embryo growth and compared the results to those obtained in the DustPy models in Fig. 8. Although there are some differences, the results in terms of the time taken to reach the pebble isolation mass or the final embryo mass usually agree within one order of magnitude. The biggest differences arise for planets introduced late, at $t_{0}=10^{6} \mathrm{yr}$. This is because the pebble predictor does not include the gas disk evolution.

\subsection{Early planet formation}

Studies of mass reservoirs of planet-forming disks infer that planet(-esimal) formation should start early (Greaves \& Rice 2011; Najita \& Kenyon 2014; Manara et al. 2018; Tychoniec et al. 2020). There is growing evidence that the planet formation process is indeed well underway in young disks (Harsono et al. 2018; Segura-Cox et al. 2020). Pebble accretion may certainly be an avenue toward fast planet formation, as young massive disks should facilitate very high pebble fluxes.

Our results show that the pebble flux is highest just after the dust aggregates at a given orbital distance reach their maximum size. Thus, if a large enough embryo forms early enough, it may benefit from this high pebble flux and reach its pebble isolation mass during the early stages of disk evolution (Tanaka \& Tsukamoto 2019). The pathway to such an early formation of massive planetesimals is not yet understood. The fastest route to planetesimal formation, the streaming instability, needs an enhanced solid-to-gas ratio to operate (Johansen et al. 2009; Bai \& Stone 2010). Enhancing their density requires the significant redistribution of solids, which may take a long time (Drążkowska et al. 2016). The cold-finger effect at the water snow line could lead to the formation of some planetesimals as early as during the disk's buildup stage (Drążkowska \& Dullemond 2018). However, the planetesimals formed via the streaming instability are most likely not large enough to accrete pebbles, and an intermediate stage of planetesimal accretion is necessary (Liu et al. 2019).

\subsection{Substructures}

In this paper, we focus on the smooth disk model. However, there is observational evidence that substructures are ubiquitous in

\footnotetext{
3 The sum in Eq. (5) may actually be omitted by using a single pebble accretion efficiency parameter calculated for the flux-averaged Stokes number and the total flux of the solids. This approach gives a very good agreement with the results presented in this paper.
} 

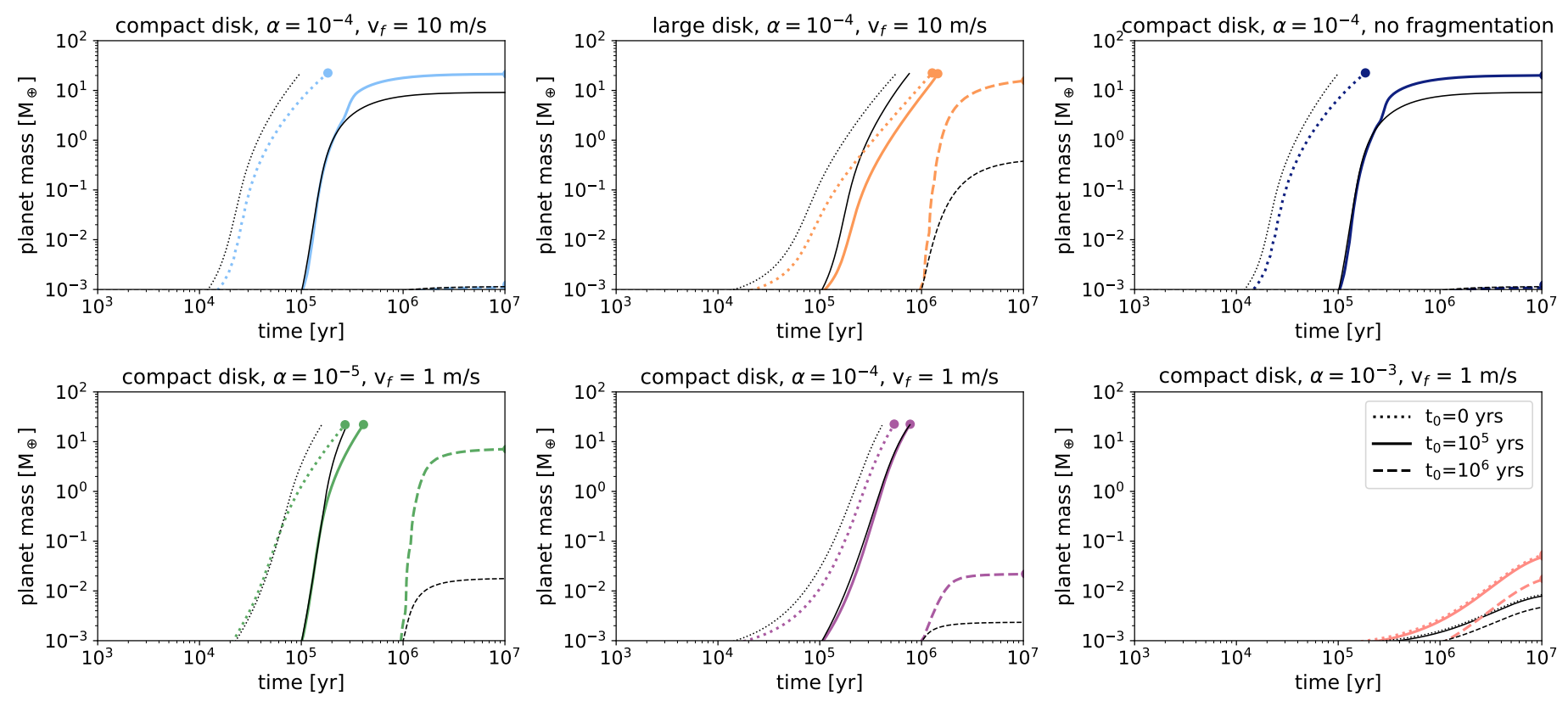

Fig. 8. Planetary embryos growth tracks obtained in various DustPy models (colored lines), as indicated by the title of each subplot and calculated using the pebble predictor results (black lines). Planetary growth is considered at 10 au with different starting times, as indicated by different line types.

protoplanetary disks (ALMA Partnership et al. 2015; Long et al. 2018; Andrews et al. 2018). Substructures are thought to modify the evolution of dust, allowing for the retention of solids over long timescales, which is consistent with observations (Pinilla et al. 2012b; Li et al. 2019b). They may be a preferential location for planetesimal and planet formation (Haghighipour \& Boss 2003; Brauer et al. 2008b). In particular, planetesimal formation may be triggered at the outer edge of a gap carved by a preexisting planet (Stammler et al. 2019; Eriksson et al. 2020; Shibaike \& Alibert 2020).

In terms of pebble flux, substructures cause the pileup of pebbles, likely speeding up local growth of planets located inside the pressure bump (Guilera et al. 2020; Morbidelli 2020). The existence of a long-lasting substructure may be a way to enable the growth of giant planets at large orbital distances. At the same time, however, overall pebble flux through the disk inward from a pressure bump would decrease as a (potentially significant) fraction of the solids are held in the substructure.

Comprehensive knowledge of the substructure and detailed dust models are necessary to calculate the resulting pebble flux. One-dimensional models of dust evolution in a pressure bump showed that dust growth can proceed to larger sizes inside a pressure bump (Brauer et al. 2008b; Pinilla et al. 2012a). Dust trapping is hindered by the diffusion and advection of small grains with the gas flow. There is a "critical dust size", which depends on the details of the bump structure, below which the grains cannot be trapped (Pinilla et al. 2016; Weber et al. 2018). This effect can, in principle, be modeled with DustPy, which includes both dust diffusion and advection with the gas flow; however, the results must be treated with caution, as recent 2D models show that the planet-induced spiral wakes may modify the dust growth pattern (Drążkowska et al. 2019). The simple flux prediction we proposed in Sect. 4 does not include diffusion and grain advection with the gas flow, and thus it is not suitable for disks with substructures. We leave the development of tools that would be useful in such disks for future work.

\section{Summary}

In this work, we studied planetary growth by pebble accretion in a protoplanetary disk without a substructure. We investigated how the most uncertain parameters - the fragmentation velocity, disk size, and turbulence strength - impact the growth rate of embryos placed at different radial locations and times during the dust evolution. We found that planetary growth strongly depends on dust evolution, and the pebble sizes and fluxes should be calculated self-consistently with the disk structure. An important byproduct of this paper is the pebble predictor, which is a publicly available script that predicts the Stokes number and flux of pebbles in any smooth protoplanetary disk model for a given fragmentation threshold velocity at a very low computational cost (see Sect. 4).

One of our conclusions is that planetary growth by pebble accretion may be extremely fast if a large embryo is formed in the disk while the pebble flux is still high. Compact disks may produce very high pebble flux, leading to the close-in embryos reaching their pebble isolation mass within $10^{4}-10^{5} \mathrm{yr}$, depending on the fragmentation speed, when the turbulence is not too strong $\left(\alpha \leq 10^{-4}\right)$. High turbulence levels universally reduce the planetary growth rate, while the effects of the fragmentation threshold and disk size are not as obvious.

Dust fragmentation keeps dust aggregates at pebble sizes, for which pebble accretion is the most efficient. Without fragmentation, dust growth would proceed over the pebble size limit, rendering the solids useless for growing planets by pebble accretion. Strong fragmentation keeps the pebbles small and decreases their drift speed, lowering pebble flux and slowing down the growth of embryos. At the same time, the low fragmentation threshold promotes the maintenance of a significant fraction of solids in the protoplanetary disk over long timescales and enables the growth of embryos that formed late.

Compact disks are favorable for the very rapid growth of close-in planets. In contrast, large disks are needed for the 
formation of giant planets on wide orbits. Even with the large disk, growing cores of giant planets outside 10 au is challenging, as the efficiency of pebble accretion drops with radial distance. One possible solution to this problem may be the existence of dust traps that could facilitate planetary growth by pebble accretion.

Acknowledgements. We thank the anonymous referee for the constructive report and Gijs Mulders for useful comments on pebble predictor. The authors acknowledge funding from the European Research Council (ERC) under the European Unions Horizon 2020 research and innovation programme under grant agreement No. 714769, the Deutsche Forschungsgemeinschaft (DFG, German Research Foundation) under Germany's Excellence Strategy - EXC-2094 390783311, and the support from the DFG Research Unit "Transition Disks" (FOR 2634/1).

\section{References}

ALMA Partnership, Brogan, C. L., Pérez, L. M., et al. 2015, ApJ, 808, L3 Andrews, S. M., Huang, J., Pérez, L. M., et al. 2018, ApJ, 869, L41 Ansdell, M., Williams, J. P., Trapman, L., et al. 2018, ApJ, 859, 21 Ataiee, S., Baruteau, C., Alibert, Y., \& Benz, W. 2018, A\&A, 615, A110 Aumatell, G., \& Wurm, G. 2014, MNRAS, 437, 690

Bai, X.-N. 2016, ApJ, 821, 80

Bai, X.-N. 2017, ApJ, 845, 75

Bai, X.-N., \& Stone, J. M. 2010, ApJ, 722, 1437

Birnstiel, T., \& Andrews, S. M. 2014, ApJ, 780, 153

Birnstiel, T., Dullemond, C. P., \& Brauer, F. 2010, A\&A, 513, A79

Birnstiel, T., Klahr, H., \& Ercolano, B. 2012, A\&A, 539, A148

Bitsch, B. 2019, A\&A, 630, A51

Bitsch, B., \& Johansen, A. 2016, A\&A, 590, A101

Bitsch, B., Lambrechts, M., \& Johansen, A. 2015, A\&A, 582, A112

Bitsch, B., Lambrechts, M., \& Johansen, A. 2018a, A\&A, 609, C2

Bitsch, B., Morbidelli, A., Johansen, A., et al. 2018b, A\&A, 612, A30

Bitsch, B., Izidoro, A., Johansen, A., et al. 2019a, A\&A, 623, A88

Bitsch, B., Raymond, S. N., \& Izidoro, A. 2019b, A\&A, 624, A109

Blum, J. 2018, Space Sci. Rev., 214, 52

Brasser, R., \& Mojzsis, S. J. 2020, Nat. Astron., 4, 492

Brauer, F., Dullemond, C. P., \& Henning, T. 2008a, A\&A, 480, 859

Brauer, F., Henning, T., \& Dullemond, C. P. 2008b, A\&A, 487, L1

Brügger, N., Alibert, Y., Ataiee, S., \& Benz, W. 2018, A\&A, 619, A174

Chambers, J. E. 2016, ApJ, 825, 63

Chambers, J. E. 2018, ApJ, 865, 30

Charnoz, S., Pignatale, F. C., Hyodo, R., et al. 2019, A\&A, 627, A50

Cridland, A. J., Pudritz, R. E., \& Birnstiel, T. 2017, MNRAS, 465, 3865

Drążkowska, J., \& Dullemond, C. P. 2018, A\&A, 614, A62

Drążkowska, J., Windmark, F., \& Okuzumi, S. 2014, IAU Symp. 310, 208

Drążkowska, J., Alibert, Y., \& Moore, B. 2016, A\&A, 594, A105

Drążkowska, J., Li, S., Birnstiel, T., Stammler, S. M., \& Li, H. 2019, ApJ, 885,

Eriksson, L. E. J., Johansen, A., \& Liu, B. 2020, A\&A, 635, A110

Flaherty, K. M., Hughes, A. M., Rosenfeld, K. A., et al. 2015, ApJ, 813, 99

Flaherty, K., Hughes, A. M., Simon, J. B., et al. 2020, ApJ, 895, 109

Gárate, M., Birnstiel, T., Drążkowska, J., \& Stammler, S. M. 2020, A\&A, 635, A 149

Greaves, J. S., \& Rice, W. K. M. 2011, MNRAS, 412, L88

Guilera, O. M., Sándor, Z., Ronco, M. P., Venturini, J., \& Miller Bertolami, M. M. 2020, A\&A, 642, A140

Gundlach, B., \& Blum, J. 2015, ApJ, 798, 34

Gundlach, B., Schmidt, K. P., Kreuzig, C., et al. 2018, MNRAS, 479, 1273

Güttler, C., Blum, J., Zsom, A., Ormel, C. W., \& Dullemond, C. P. 2010, A\&A, 513, A56

Haghighipour, N., \& Boss, A. P. 2003, ApJ, 583, 996

Harsono, D., Bjerkeli, P., van der Wiel, M. H. D., et al. 2018, Nat. Astron., 2, 646

Hernández, J., Hartmann, L., Megeath, T., et al. 2007, ApJ, 662, 1067

Ida, S., \& Makino, J. 1993, Icarus, 106, 210

Ida, S., Guillot, T., \& Morbidelli, A. 2016, A\&A, 591, A72

Izidoro, A., Bitsch, B., Raymond, S. N., et al. 2019, A\&A, submitted

Johansen, A., \& Bitsch, B. 2019, A\&A, 631, A70

Johansen, A., Youdin, A., \& Mac Low, M.-M. 2009, ApJ, 704, L75

Johansen, A., Mac Low, M.-M., Lacerda, P., \& Bizzarro, M. 2015, Sci. Adv., 1 , 1500109

Johansen, A., Ida, S., \& Brasser, R. 2019, A\&A, 622, A202

Klahr, H., \& Schreiber, A. 2020, ApJ, 901, 54

Kokubo, E., \& Ida, S. 1996, Icarus, 123, 180

Kokubo, E., \& Ida, S. 1998, Icarus, 131, 171
Kokubo, E., \& Ida, S. 2002, ApJ, 581, 666

Kretke, K. A., \& Levison, H. F. 2014, AJ, 148, 109

Laibe, G. 2014, MNRAS, 437, 3037

Lambrechts, M., \& Johansen, A. 2012, A\&A, 544, A32

Lambrechts, M., \& Johansen, A. 2014, A\&A, 572, A107

Lambrechts, M., Johansen, A., \& Morbidelli, A. 2014, A\&A, 572, A35

Lambrechts, M., Morbidelli, A., Jacobson, S. A., et al. 2019, A\&A, 627, A83

Lesur, G., Kunz, M. W., \& Fromang, S. 2014, A\&A, 566, A56

Levison, H. F., Kretke, K. A., \& Duncan, M. J. 2015a, Nature, 524, 322

Levison, H. F., Kretke, K. A., Walsh, K. J., \& Bottke, W. F. 2015b, Proc. Natl. Acad. Sci., 112, 14180

Levison, H. F., Thommes, E., \& Duncan, M. J. 2010, AJ, 139, 1297

Li, R., Youdin, A. N., \& Simon, J. B. 2019a, ApJ, 885, 69

Li, Y.-P., Li, H., Ricci, L., et al. 2019b, ApJ, 878, 39

Liu, B., \& Ormel, C. W. 2018, A\&A, 615, A138

Liu, B., Ormel, C. W., \& Johansen, A. 2019, A\&A, 624, A114

Long, F., Herczeg, G. J., Harsono, D., et al. 2019, ApJ, 882, 49

Long, F., Pinilla, P., Herczeg, G. J., et al. 2018, ApJ, 869, 17

Lynden-Bell, D., \& Pringle, J. E. 1974, MNRAS, 168, 603

Manara, C. F., Morbidelli, A., \& Guillot, T. 2018, A\&A, 618, L3

Matsumura, S., Brasser, R., \& Ida, S. 2017, A\&A, 607, A67

Maury, A. J., André, P., Testi, L., et al. 2019, A\&A, 621, A76

Meru, F., Geretshauser, R. J., Schäfer, C., Speith, R., \& Kley, W. 2013, MNRAS, 435, 2371

Morbidelli, A. 2020, A\&A, 638, A1

Mori, S., Bai, X.-N., \& Okuzumi, S. 2019, ApJ, 872, 98

Musiolik, G., \& Wurm, G. 2019, ApJ, 873, 58

Najita, J. R., \& Kenyon, S. J. 2014, MNRAS, 445, 3315

Ndugu, N., Bitsch, B., \& Jurua, E. 2018, MNRAS, 474, 886

Ogihara, M., \& Hori, Y. 2020, ApJ, 892, 124

Ogihara, M., Morbidelli, A., \& Guillot, T. 2015, A\&A, 578, A36

Okuzumi, S., Tanaka, H., Kobayashi, H., \& Wada, K. 2012, ApJ, 752, 106

Ormel, C. W. 2017, Astrophys. Space Sci. Lib., 445, 197

Ormel, C. W., \& Cuzzi, J. N. 2007, A\&A, 466, 413

Ormel, C. W., \& Klahr, H. H. 2010, A\&A, 520, A43

Ormel, C. W., \& Liu, B. 2018, A\&A, 615, A178

Ormel, C. W., Dullemond, C. P., \& Spaans, M. 2010, ApJ, 714, L103

Pinilla, P., Benisty, M., \& Birnstiel, T. 2012a, A\&A, 545, A81

Pinilla, P., Birnstiel, T., Ricci, L., et al. 2012b, A\&A, 538, A114

Pinilla, P., Klarmann, L., Birnstiel, T., et al. 2016, A\&A, 585, A35

Pinte, C., Dent, W. R. F., Ménard, F., et al. 2016, ApJ, 816, 25

Powell, D., Murray-Clay, R., Pérez, L. M., Schlichting, H. E., \& Rosenthal, M. 2019, ApJ, 878, 116

Ricci, L., Testi, L., Natta, A., \& Brooks, K. J. 2010, A\&A, 521, A66

Sato, T., Okuzumi, S., \& Ida, S. 2016, A\&A, 589, A15

Schäfer, U., Yang, C.-C., \& Johansen, A. 2017, A\&A, 597, A69

Segura-Cox, D. M., Schmiedeke, A., Pineda, J. E., et al. 2020, Nature, 586, 228

Shibaike, Y., \& Alibert, Y. 2020, A\&A, 644, A81

Stammler, S. M., Drążkowska, J., Birnstiel, T., et al. 2019, ApJ, 884, L5

Steinpilz, T., Teiser, J., \& Wurm, G. 2019, ApJ, 874, 60

Tamfal, T., Drążkowska, J., Mayer, L., \& Surville, C. 2018, ApJ, 863, 97

Tanaka, Y. A., \& Tsukamoto, Y. 2019, MNRAS, 484, 1574

Teague, R., Guilloteau, S., Semenov, D., et al. 2016, A\&A, 592, A49

Teague, R., Henning, T., Guilloteau, S., et al. 2018, ApJ, 864, 133

Thommes, E. W., Duncan, M. J., \& Levison, H. F. 2002, AJ, 123, 2862

Thommes, E. W., Duncan, M. J., \& Levison, H. F. 2003, Icarus, 161, 431

Tobin, J. J., Sheehan, P. D., Megeath, S. T., et al. 2020, ApJ, 890, 130

Trapman, L., Rosotti, G., Bosman, A. D., Hogerheijde, M. R., \& van Dishoeck, E. F. 2020, A\&A, 640, A5

Tychoniec, Ł., Manara, C. F., Rosotti, G. P., et al. 2020, A\&A, 640, A19

Venturini, J., Guilera, O. M., Haldemann, J., Ronco, M. P., \& Mordasini, C. 2020a, A\&A, 643, L1

Venturini, J., Guilera, O. M., Ronco, M. P., \& Mordasini, C. 2020b, A\&A, 644, A174

Villenave, M., Ménard, F., Dent, W. R. F., et al. 2020, A\&A, 642, A164

Visser, R. G., \& Ormel, C. W. 2016, A\&A, 586, A66

Wada, K., Tanaka, H., Suyama, T., Kimura, H., \& Yamamoto, T. 2011, ApJ, 737, 36

Ward, W. R. 1997, Icarus, 126, 261

Weber, P., Benítez-Llambay, P., Gressel, O., Krapp, L., \& Pessah, M. E. 2018, ApJ, 854, 153

Weidenschilling, S. J. 1977, MNRAS, 180, 57

Wetherill, G. W., \& Stewart, G. R. 1989, Icarus, 77, 330

Wilner, D. J., D’Alessio, P., Calvet, N., Claussen, M. J., \& Hartmann, L. 2005, ApJ, 626, L109

Wimarsson, J., Liu, B., \& Ogihara, M. 2020, MNRAS, 496, 3314

Zormpas, A., Picogna, G., Ercolano, B., \& Kley, W. 2020, A\&A, 638, A97 